\title{
Horseshoes in 3D equations with applications to Lotka-Volterra systems
}

\author{
Alfonso Ruiz-Herrera and Fabio Zanolin
}

\begin{abstract}
We discuss a geometric configuration for a class of homeomorphisms in $\mathbb{R}^{3}$ producing the existence of infinitely many periodic points as well a complex dynamics due to the presence of a topological horseshoe. We also show that such a class of homeomorphisms appears in the classical Lotka-Volterra system.
\end{abstract}

Mathematics Subject Classification. Primary 34C25; Secondary 37C25, $37 \mathrm{D} 45$.

Keywords. Complex dynamics, Poincaré's map, Topological horseshoes, Periodic solutions, Three dimensional predator-prey equations, Switching systems.

\section{Introduction}

Systems of ordinary differential equations of the form

$$
x^{\prime}=\mathscr{X}(t, x),
$$

where $\mathscr{X}: \mathbb{R} \times \mathbb{R}^{N} \rightarrow \mathbb{R}^{N}$ is a vector field $T$-periodic in the $t$-variable, arise in large number of different situations, such as mechanical systems subject to periodic disturbances, biological models concerning the interaction of species in a seasonally varying environment, electric circuit theory, dynamics of fluids, etc. In this setting, the study of existence and multiplicity of periodic solutions, as well as the search for solutions with more complex behavior, plays a crucial role in the investigations about Eq. (1.1). Accordingly, a great deal of research

ERC Starting Investigator Grant No. 259559, EPIDELAY. The research of A. R.-H. was performed in the framework of the TÁMOP 4.2.4.A/2-11-1-2012-0001 "National Excellence Programme Elaborating and operating an inland student and researcher personal support system convergence programme Control tools in biology, which is subsidized by the European Union and Hungary and co-financed by the European Social Fund. The research of F. Z. was performed under the auspices of the Gruppo Nazionale per l'Analisi Matematica, la Probabilità e le loro Applicazioni (GNAMPA) of the Istituto Nazionale di Alta Matematica (INdAM). 
has been performed in such a direction, both from the point of view of studying the dynamical system properties associated with the solutions, and in the development of new functional analytic tools which provide the existence and multiplicity of fixed points for some operator equations. See [10,20,21,25,32] for important contributions and basic references about the subject.

This paper is devoted to the search of periodic solutions and chaotic dynamics for the Poincaré map associated with Eq. (1.1) in the three-dimensional case. In the literature one can find many different concepts of complex dynamics or "chaotic motions" for a map. The definition of chaos which we are going to use is manly motivated by Smale's expository article [37]. Intuitively, we can say that a chaotic phenomenon occurs if it is possible to reproduce, within the system and varying the initial conditions, all the possible outcomes of a coin-flipping experiment. To describe more formally this situation we consider a map $f$ acting on a metric space $X, k \geq 2$ nonempty compact disjoint sets $H_{0}, \ldots, H_{k-1}$ and a compact set $\Lambda \subseteq \cup_{i=0}^{k-1} H_{i}$ which is invariant under $f$, that is $f(\Lambda)=\Lambda$. The itinerary of each point $x \in \Lambda$, under the action of $f$ is expressed by means of the Picard sequence $y_{n+1}=f\left(y_{n}\right)$, with $y_{0}=x$, or, in other terms, by the trajectory $\left(f^{n}(x)\right)_{n}$. Such trajectory is coded as a sequence of symbols $\left(s_{n}\right)_{n}$ with $s_{n} \in\{0, \ldots, k-1\}$, where $s_{n}=j$ means that $f^{n}(x) \in H_{j}$ with $j=0, \ldots, k-1$. Representing the orbits of a map $f$ by sequences of symbols is a widely explored point of view, dating back to Hadamard, Birkhoff, Morse-Hedlund, Cartwright-Littlewood and Levinson (see [28]). In order to present a simpler situation, let us confine ourselves for a moment to the case of only two symbols, say 0 and 1 . In this framework, a fundamental model is given by the so-called Bernoulli shift which is a map $\sigma$ acting on the set $\Sigma_{2}=\{0,1\}^{\mathbb{Z}}$ of bi-infinite sequences of two symbols and defined as

$$
\sigma:\left(s_{n}\right)_{n \in \mathbb{Z}} \mapsto\left(s_{n}^{\prime}\right)_{n \in \mathbb{Z}}, \quad \text { with } s_{n}^{\prime}=s_{n+1} .
$$

According to the above procedure, a strategy to prove the presence of complex dynamics for a given map $f$, is to link the behavior of $f$ on the invariant set $\Lambda$ to that of the Bernoulli shift $\sigma$ on $\Sigma_{2}$. The connection between $\left.f\right|_{\Lambda}$ and $\sigma$ is formally expressed by a continuous map $g: \Lambda \rightarrow \Sigma_{2}$ which makes the diagram

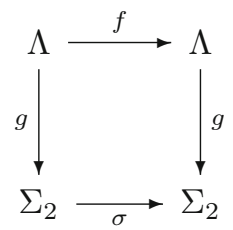

commutative. In some examples the map $g$ is a homeomorphism between compact spaces (recall that $\Sigma_{2}$ is a compact space endowed with a standard metric for the product topology). In such a case we say that $f$ is conjugate to $\sigma$. When this situation occurs, $\sigma$ reproduces the dynamics of $f$ in $\Lambda$ and all the topological properties of $\sigma$, like transitivity and density of the periodic points, are inherited by $f$.

In the sixties [35,36], Smale provided a geometrical and very useful description of a diffeomorphism $f$ on manifolds having an invariant set on which 


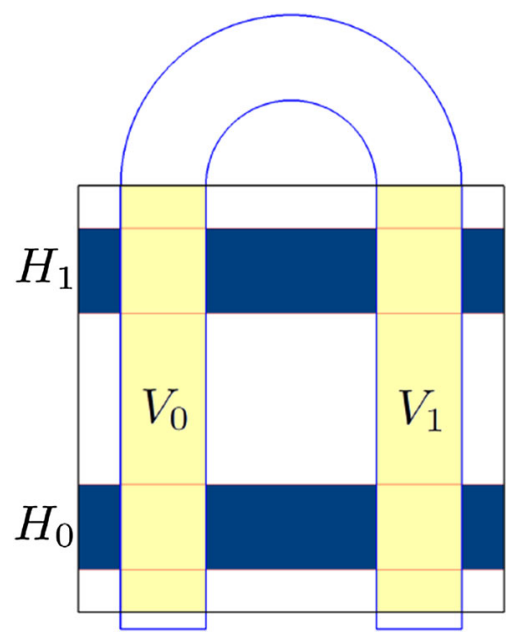

FiguRE 1. A typical image representing the Smale horseshoe. The horizontal strips $H_{0}$ and $H_{1}$ are mapped to the vertical strips $V_{0}$ and $V_{1}$

$f$ is conjugate to $\sigma$. In the planar setting the map can be described as a function which contracts a square $Q=[0,1]^{2}$ in the $x$-direction, expands it in the $y$ direction and further twists it around, in such a manner that $f(Q)$ looks as a horseshoe over $Q$ (see Fig. 1). For more details about this construction and various applications of this prototypical example, we refer to some classical books like $[11,28]$.

The theory of Smale horseshoes has been successfully applied to some specific situations, leading to rigorous proofs of the existence of chaotic dynamics. These applications are not restricted to the planar case since they can be developed in the higher dimensional setting too, for instance, in the Mel'nikov or the Sil'nikov configurations or in other geometrical constructions (see [11]). In general, however, such tools are not easy to apply. This fact has motivated several authors to propose different approaches in which some technical assumptions related to the classical Smale horseshoe, are, at a certain extent, relaxed to suitable conditions of more topological nature. Such a point of view was already present in the pioneering works of Smale and Alekseev and, more explicitly, in the Conley and Moser approach in [28, Chapter 3]. More recent contributions in this topological direction, using different tools, like Conley index theory, homological methods, topological degree, have been obtained by Kennedy, Yorke, Gidea, Mischaikow, Mrozek, Srzednicki, Wójcik, Zgliczyński and their collaborators (see, for instance, [6,18,38,41-43] and the references therein). A possible way to develop the underlying geometry for the Smale horseshoe was proposed by Burns and Weiss in [4] as a method by which one proves that a set is mapped across itself in the appropriate way. Figure 2 shows a simple case in which a rectangle is crossed by a horseshoe-like set 


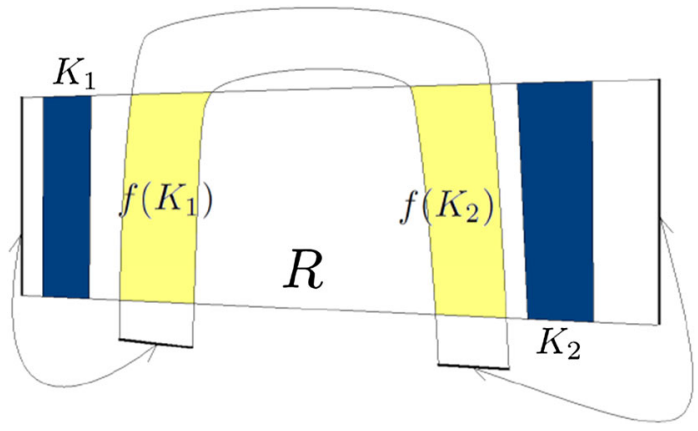

FiguRE 2. Example of a bad crossing. In this picture we have a rectangular domain $R$ which is transformed by a homeomorphism $f$ into a horseshoe type figure. Observe that there is no fixed point for $f$ in $R$. Furthermore, for each $x \in R$, we have $f^{2}(x) \notin R$ and therefore $R$ does not contain any nonempty invariant set

in a wrong manner. Such kind of questions are also raised and discussed by Kennedy, Koçak and Yorke in the last part of the article [16].

In the present work we consider a topological approach for detecting the presence of horseshoe type phenomena for a map $\phi$ defined on a domain homeomorphic to the unit cube $\mathcal{C}:=[0,1]^{3}$. The "appropriate crossing" of $\phi(\mathcal{C})$ along $\mathcal{C}$ is technically expressed by a property that we name stretching along the paths. Roughly speaking it means that any path $\zeta$ contained in $\mathcal{C}$ and joining two opposite faces of $\mathcal{C}$, contains at least two sub-paths which are stretched by $f$ along $\mathcal{C}$ and connect the same faces as $\zeta$ (see Sect. 2 for the formal definitions and more details). Our method can be viewed as a variant of the theory of topological horseshoes developed by Kennedy and Yorke [17].

As in other topological methods generalizing Smale's construction, also in our approach we are able to obtain a compact invariant set $\Lambda$ and a commutative diagram like in (1.2), with $g$ continuous and surjective (but not necessarily one-to-one), which, technically speaking, corresponds to the case of a semiconjugation. Even if this is a weaker form of chaos [as we cannot ensure in general that the map $g$ in (1.2) is a bijection], the semiconjugation to the Bernoulii shift is an important feature of any reasonable definition of chaotic dynamics, as pointed out by Block and Coppel in [3].

In general, as shown by classical examples $[6,17]$, the surjectivity of $g$ does not ensure the presence of periodic points for the given map $f$. In contrast with this fact, our results provide the existence of infinitely many periodic points in the invariant set. More precisely we can prove that, for any periodic sequence $c=\left(s_{n}\right)_{n \in \mathbb{Z}} \in \Sigma_{2}$ the counter-image $g^{-1}(c)$ contains at least one periodic point having the same period of the sequence $c$. This makes our approach particularly suitable to study the periodic problem associated with (1.1), for instance, in connection to the search of subharmonic solutions. In conclusion we can say that, in relation to other definitions of chaotic dynamics considered 
in the literature, our notion is consistent with the concept of chaos in the cointossing sense $[19,37]$ but with a special emphasis toward the existence and multiplicity of periodic (harmonic and subharmonic) solutions. Similar kind of chaotic dynamics have been detected under different topological approaches (see, for instance, $[6,38,41-43])$.

As a concrete application of our method we study the classical LotkaVolterra model for two predators $\left(x_{2}\right.$ and $\left.x_{3}\right)$ and one prey $\left(x_{1}\right)$ in a periodic environment. More in detail, we consider the system

$$
\left\{\begin{array}{l}
x_{1}^{\prime}=x_{1}\left(\alpha(t)-a_{11}(t) x_{1}-a_{12}(t) x_{2}-a_{13}(t) x_{3}\right) \\
x_{2}^{\prime}=x_{2}\left(\beta(t)+a_{21}(t) x_{1}-a_{22}(t) x_{2}-a_{23}(t) x_{3}\right) \\
x_{3}^{\prime}=x_{3}\left(\gamma(t)+a_{31}(t) x_{1}-a_{32}(t) x_{2}-a_{33}(t) x_{3}\right)
\end{array}\right.
$$

where all the coefficients are $T$-periodic and $a_{i j}(t) \geq 0$. Although we focus our attention on system (1.3), our approach enables us to consider different models for the interaction, like Holling type, Beddington-DeAngelis or Gompertz.

Lotka-Volterra equations have been extensively studied from different perspectives, dealing with problems such as permanence, stability, extinction, existence and multiplicity of periodic solutions, or invading species (see $[1,7,8,22,23,26,39])$. Fewer results, however, concern the presence of chaotic dynamics for these equations, especially from an analytic point of view.

The application of our general topological method to system (1.3) is based on a splitting of the associated Poincaré map $\phi$ as a composition of three functions $f, g, h$. For each of these functions we assume some geometrical conditions which are a direct consequence of some hypotheses on the coefficients of (1.3). In order to express the topological properties required for such maps in the clearest manner, we first present a prototypical situation where the essential features can be easily described.

To conclude this Introduction, we remark that developments of the theory of topological horseshoes have been applied in the planar context or to some higher dimensional Hamiltonians (see [2,5,29,41] and the references therein). Up to now, fewer applications in higher dimension and, especially in the nonHamiltonian setting, like in case of (1.3), are available.

The plan of the paper is the following. In Sect. 2 we recall from [34] our main definitions and abstract results which are applied in the paper. Subsequently, we illustrate a three-dimensional geometry producing a horseshoe type dynamics for a homeomorphism which can be split as the composition of three maps. Section 4 is devoted to the presentation of our main result (Theorem 4.2) for a classical Lotka-Volterra system describing the interaction of two predators and one prey in a periodic environment. The proof with all the details is given the last section.

\section{Topological results}

In this section we collect the main definitions and the abstract results which are needed for the proofs of our theorems. We refer the interested reader to [34] for more details and [30,31] for related general results. Our method deals 
with continuous maps acting on suitable sets named oriented cylinders. By an oriented cylinder of $\mathbb{R}^{N}$ we mean a set which is homeomorphic to the product of a closed ball in $\mathbb{R}^{N-1}$ with a compact interval and where we have put in evidence two opposite faces. Sets of this form have been also considered in similar approaches by other authors as $[2,43]$ and are also called $(u, v)$-sets or "windows", in the literature. Since our results are stable by homeomorphisms deforming the domain, we can consider, as a generic example, a (solid) cylinder of the form

$$
\mathscr{C}=B_{R} \times[0,1],
$$

where $B_{R}$ is the closed ball in $\mathbb{R}^{N-1}$ with center at the origin and radius $R>0$. The base and the top of the cylinder will be denoted by $\mathscr{C}_{0}^{-}=B_{R} \times\{0\}$ and $\mathscr{C}_{1}^{-}=B_{R} \times\{1\}$. Formally, an oriented cylinder is the pair

$$
\widetilde{\mathscr{C}}:=\left(\mathscr{C}, \mathscr{C}^{-}\right)
$$

with

$$
\mathscr{C}^{-}:=\mathscr{C}_{0}^{-} \cup \mathscr{C}_{1}^{-}
$$

The sets $\mathscr{C}_{0}^{-}$and $\mathscr{C}_{1}^{-}$are the two components of $\mathscr{C}^{-}$.

Let $K$ be a (nonempty) compact subset of $\mathscr{C}$ and let $\Phi: \mathscr{C} \rightarrow \mathbb{R}^{N}$ be a continuous map. We say that the pair $(\Phi, K)$ has the stretching along the paths property with respect to $\mathscr{C}$ and write

$$
(\Phi, K): \widetilde{\mathscr{C}} \cong \widetilde{\mathscr{C}}
$$

if, for every (continuous) path $\zeta:\left[t_{0}, t_{1}\right] \rightarrow \mathscr{C}$ such that $\zeta\left(t_{0}\right) \in \mathscr{C}_{0}^{-}$and $\zeta\left(t_{1}\right) \in \mathscr{C}_{1}^{-}\left(\right.$or $\zeta\left(t_{0}\right) \in \mathscr{C}_{1}^{-}$and $\left.\zeta\left(t_{1}\right) \in \mathscr{C}_{0}^{-}\right)$, there exists a subinterval $\left[s_{0}, s_{1}\right]$ of $\left[t_{0}, t_{1}\right]$ for which we have that

$\zeta(t) \in K, \quad$ for all $t \in\left[s_{0}, s_{1}\right]$;

$\Phi(\zeta(t)) \in \mathscr{C}$, for all $t \in\left[s_{0}, s_{1}\right]$;

$\Phi\left(\zeta\left(s_{0}\right)\right)$ and $\Phi\left(\zeta\left(s_{1}\right)\right)$ belong to different components of $\mathscr{C}^{-}$.

If $K_{0}$ and $K_{1}$ are two (nonempty) compact and disjoint subsets of $\mathscr{C}$ such that

$$
\left(\Phi, K_{i}\right): \widetilde{\mathscr{C}} \cong \widetilde{\mathscr{C}}, \text { for } i=0,1,
$$

then we also write

$$
\left(\Phi, K_{0}, K_{1}\right): \widetilde{\mathscr{C}} \cong \widetilde{\mathscr{C}} .
$$

The above definitions easily extend to the case in which the stretching property is satisfied between two different (oriented) cylinders. In this framework, the following result holds [34, Theorem 2.1].

Theorem 2.1. Let $\widetilde{\mathscr{C}}, K_{0}$ and $K_{1}$ be as above and let $\Phi: \mathscr{C} \rightarrow \mathbb{R}^{N}$ be a homeomorphism (onto its image). Suppose that $\left(\Phi, K_{0}, K_{1}\right): \widetilde{\mathscr{C}} \bumpeq \widetilde{\mathscr{C}}$. Then $\Phi$ is chaotic.

The term "chaotic" in the above theorem refers to dynamics of cointossing type as described in the Introduction. More specifically, the following situation occurs: There exists a compact set $\Lambda \subset K_{0} \cup K_{1}$ which is invariant 
under $\Phi$ and such that for any two-sided sequence $\left(s_{n}\right)_{n \in \mathbb{Z}} \in \Sigma_{2}$ there exists a point $w \in \Lambda$ with

$$
\Phi^{n}(w) \in K_{s_{n}}, \quad \text { for each } n \in \mathbb{Z} .
$$

Moreover, if $\left(s_{n}\right)_{n \in \mathbb{Z}}$ is a $k$-periodic sequence (that is $s_{i+k}=s_{i}$ for some $k \geq 1$ and all $i \in \mathbb{Z}$ ) there exists at least one $k$-periodic point of $\Phi$ satisfying (2.1). In this setting, $\Lambda$ can be chosen so that the set of periodic points Per $\Phi$ is dense in $\Lambda$ and $\left.\Phi\right|_{\Lambda}$ is semiconjugate to the Bernoulli shift on $\Sigma_{2}$ (see [27] for a complete list of the properties).

In view of the applications, we have intentionally stated our results for the case of a map $\Phi$ which is a homeomorphism. The theory can be generalized for continuous maps.

\section{A prototypical example}

In order to show how Theorem 2.1 can be applied to a three dimensional ODE system, we describe a model example in which the map $\phi$ may be split as the composition of three functions. To make our assumptions more transparent, we restrict our presentation to a simpler situation, namely the case where the domain is a cube. The same construction can be adapted to more general frameworks in which the pertinent domains of the involved maps are compact sets homeomorphic to the unit cube.

Consider in $\mathbb{R}^{3}$ the set $R:=[0,1]^{3}$ and its sub-cube $R_{0}:=[1 / 4,3 / 4]^{2} \times$ $[0,1]$. Let $\phi: \mathbb{R}^{3} \rightarrow \mathbb{R}^{3}$ be a map which can be expressed as the composition of three homeomorphisms as

$$
\phi=h \circ g \circ f .
$$

On such functions the following assumptions are made.

$\left(H_{f}\right)$ There exist two compact disjoint subsets $K_{0}$ and $K_{1}$ of $R$ such that for any path $\zeta=\left(\zeta_{1}, \zeta_{2}, \zeta_{3}\right):\left[t_{0}, t_{1}\right] \rightarrow R$ with $\zeta_{2}\left(t_{0}\right)=0$ and $\zeta_{2}\left(t_{1}\right)=1$, there exist two subintervals $\left[s_{1}^{\prime}, s_{1}^{\prime \prime}\right],\left[s_{2}^{\prime}, s_{2}^{\prime \prime}\right] \subset\left[t_{0}, t_{1}\right]$ such that for all $\theta \in\left[s_{j}^{\prime}, s_{j}^{\prime \prime}\right](j=1,2)$ it holds that $\zeta(\theta) \in K_{j}$ and, moreover, $f(\zeta(\theta)) \in R$, with $f_{1}\left(\zeta\left(s_{j}^{\prime}\right)\right)=0$ and $f_{1}\left(\zeta\left(s_{j}^{\prime \prime}\right)\right)=1$ (or, viceversa).

$\left(H_{g}\right)$ For any path $\zeta=\left(\zeta_{1}, \zeta_{2}, \zeta_{3}\right):\left[t_{0}, t_{1}\right] \rightarrow R$ with $\zeta_{1}\left(t_{0}\right)=0$ and $\zeta_{1}\left(t_{1}\right)=1$, there exists a subinterval $\left[s^{\prime}, s^{\prime \prime}\right] \subset\left[t_{0}, t_{1}\right]$ such that $g(\zeta(\theta)) \in R_{0}$ for all $\theta \in\left[s^{\prime}, s^{\prime \prime}\right]$ with $g_{3}\left(\zeta\left(s^{\prime}\right)\right)=0$ and $g_{3}\left(\zeta\left(s^{\prime \prime}\right)\right)=1$ (or, viceversa).

$\left(H_{h}\right) h_{1}\left(R_{0}\right), h_{3}\left(R_{0}\right) \subset[0,1]$ and, moreover,

$$
\begin{aligned}
& \max h_{2}\left(x_{1}, x_{2}, 0\right)<0, \quad \forall\left(x_{1}, x_{2}, 0\right) \in R_{0} \\
& \min h_{2}\left(x_{1}, x_{2}, 1\right)>1, \quad \forall\left(x_{1}, x_{2}, 1\right) \in R_{0},
\end{aligned}
$$

or

$$
\begin{aligned}
& \min h_{2}\left(x_{1}, x_{2}, 0\right)>1, \quad \forall\left(x_{1}, x_{2}, 0\right) \in R_{0} \\
& \max h_{2}\left(x_{1}, x_{2}, 1\right)<0, \quad \forall\left(x_{1}, x_{2}, 1\right) \in R_{0} .
\end{aligned}
$$

The following result is a direct consequence of Theorem 2.1. The straightforward proof is omitted. 


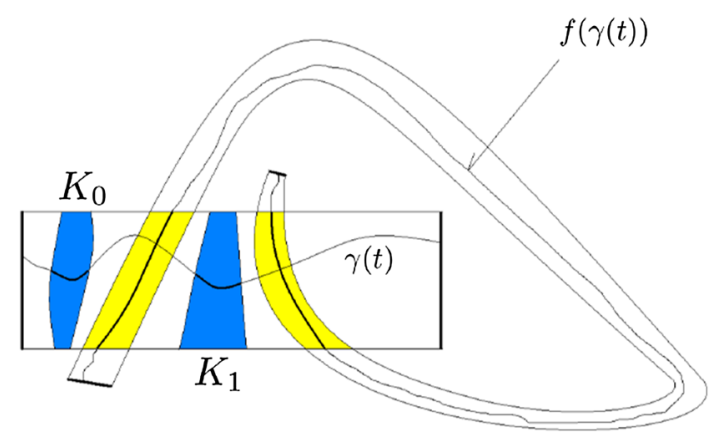

FiguRE 3. A pictorial description of condition $\left(H_{f}\right)$. Notice that $\left(H_{f}\right)$ refers to a three-dimensional situation. For simplicity we have drawn a projection in the $x_{1} x_{2}$-plane. We have used a different aspect ratio, so that the unit square looks as a rectangle. Comparing this configuration with that of Fig. 2, we see that we are in presence of a "bad crossing". This is not in contradiction with the Smale horseshoe construction since $\left(H_{f}\right)$ is only one of the three steps that we need in order to obtain the correct geometry for the map $\phi$

Theorem 3.1. Under the assumptions $\left(H_{f}\right),\left(H_{g}\right)$ and $\left(H_{h}\right)$, the map $\phi$ is chaotic on the set $R$.

The geometry associated with the map $f$ apparently reminds that of a Smale's horseshoe (see Fig. 3). However, the way in which $f(R)$ crosses the domain $R$ may not provide such a dynamical behavior. Indeed, for a map $f$ as depicted in Fig. 3 we may not have even fixed points.

\section{Statement of the main results}

In this section we focus our attention on a predator-prey type Lotka-Volterra system of the form

$$
\left\{\begin{array}{l}
x_{1}^{\prime}=x_{1}\left(\alpha(t)-a_{11}(t) x_{1}-a_{12}(t) x_{2}-a_{13}(t) x_{3}\right) \\
x_{2}^{\prime}=x_{2}\left(\beta(t)+a_{21}(t) x_{1}-a_{22}(t) x_{2}-a_{23}(t) x_{3}\right) \\
x_{3}^{\prime}=x_{3}\left(\gamma(t)+a_{31}(t) x_{1}-a_{32}(t) x_{2}-a_{33}(t) x_{3}\right)
\end{array}\right.
$$

where all the coefficients are $T$-periodic and $a_{i j}(t) \geq 0$.

In our model the variables $x_{2}$ and $x_{3}$ refer to populations predating the prey $x_{1}$. We take into account also the presence of inter-competition between $x_{2}$ and $x_{3}$ and intra-competition in each species. The (periodic) dependence on time of the coefficients of system (4.1) is introduced in order to model the seasonal effects of the environment. In many ecological situations, such a dependence is expressed as a switching between two or more regimes, where each regime is described by an autonomous system. In mathematical biology these equations are known as systems with seasonal succession [9,12,13]. For instance, these systems naturally appear when the behavior of some species 
alternates between different states (like migration, hibernation, or breeding periods), see $[9,12-15,24]$.

Our aim is to prove the presence of chaotic dynamics for the Poincaré map associated with (4.1). Recall that the Poincaré map is defined as

$$
\begin{aligned}
\Phi: \mathbb{R}_{+}^{3} \longrightarrow \mathbb{R}_{+}^{3} & :=\left\{\left(x_{1}, x_{2}, x_{3}\right): x_{i} \geq 0, \quad \text { for } i=1,2,3\right\} \\
p & \mapsto x(T ; p),
\end{aligned}
$$

where $x(t ; p)$ is the maximal solution of the system with initial condition at $p$. To this end, as a first step, we consider the following class of equations describing seasonal succession

$$
\begin{gathered}
\left\{\begin{array} { l } 
{ x _ { 1 } ^ { \prime } = x _ { 1 } ( \alpha ^ { ( 1 ) } - a _ { 1 2 } ^ { ( 1 ) } x _ { 2 } ) } \\
{ x _ { 2 } ^ { \prime } = x _ { 2 } ( - \beta ^ { ( 1 ) } + a _ { 2 1 } ^ { ( 1 ) } x _ { 1 } ) } \\
{ x _ { 3 } ^ { \prime } = 0 }
\end{array} \text { for all } t \in \left[n T, n T+T_{1}[, n \in \mathbb{Z}\right.\right. \\
\left\{\begin{array} { l } 
{ x _ { 1 } ^ { \prime } = x _ { 1 } ( \alpha ^ { ( 2 ) } - a _ { 1 3 } ^ { ( 2 ) } x _ { 3 } ) } \\
{ x _ { 2 } ^ { \prime } = x _ { 2 } ( \beta ^ { ( 2 ) } - x _ { 2 } ) } \\
{ x _ { 3 } ^ { \prime } = x _ { 3 } ( - \gamma ^ { ( 2 ) } + a _ { 3 1 } ^ { ( 2 ) } x _ { 1 } ) }
\end{array} \text { for all } t \in \left[n T+T_{1}, n T+T_{1}+T_{2}[, n \in \mathbb{Z}\right.\right. \\
\left\{\begin{array}{l}
x_{1}^{\prime}=0 \\
x_{2}^{\prime}=x_{2}\left(\beta^{(3)}-x_{3}\right) \\
x_{3}^{\prime}=0
\end{array}\right.
\end{gathered}
$$

where all the parameters are strictly positive. For convenience we introduce the notation $(\mathbf{S})$ to denote the above $T$-periodic system made by (4.2)-(4.4) and set

$$
T_{3}:=T-T_{1}-T_{2}
$$

In system (S) we perform a switching among three different seasons of length $T_{1}, T_{2}$ and $T_{3}$. Accordingly, the Poincaré map $\Phi$ associated with $(\mathbf{S})$ can be expressed as

$$
\Phi=h \circ g \circ f,
$$

where $f, g$ and $h$ are the Poincaré maps for systems (4.2)-(4.4) at the times $T_{1}, T_{2}$ and $T_{3}$, respectively. By the special form of $(\mathbf{S})$ it easily follows that $\Phi$ is defined on the whole positive cone $\mathbb{R}_{+}^{3}$.

Now we are in a position to present our main result for $(\mathbf{S})$.

Theorem 4.1. With respect to the parameters in $(\mathbf{S})$, assume that

$$
2 \beta^{(2)}<\frac{\alpha^{(1)}}{a_{12}^{(1)}} .
$$

Then there exist $\gamma_{2}^{*}, T_{1}^{*}, T_{2}^{*}=T_{2}^{*}\left(\gamma^{(2)}\right)$ and $T_{3}^{*}$ so that if $\gamma^{(2)}>\gamma_{2}^{*}$ and $T_{i}>T_{i}^{*}$ (for $i=1,2,3)$, the Poincaré map associated with $(\mathbf{S})$ is chaotic. 
Remark 4.1. In the proof we estimate $\gamma_{2}^{*}, T_{1}^{*}, T_{2}^{*}=T_{2}^{*}\left(\gamma^{(2)}\right)$ and $T_{3}^{*}$ depending on the coefficients of the equations. The compact invariant set $\Lambda$ where the Poincaré map $\Phi$ exhibits a chaotic behavior is contained in a suitable cylindrical set which is constructed inside the interior int $\left(\mathbb{R}_{+}^{3}\right)$ of the positive cone. Consequently, by the invariance of the boundary of $\mathbb{R}_{+}^{3}$ for all systems of the form (4.1), we also know that the corresponding complex solutions lie $\operatorname{in} \operatorname{int}\left(\mathbb{R}_{+}^{3}\right)$.

It is easy to check that system $(\mathbf{S})$ is a particular case of the LotkaVolterra system (4.1). For instance, $\alpha(t)$ is the function defined stepwise as $\alpha(t)=\alpha^{(1)}$ if $t \in\left[0, T_{1}\left[\right.\right.$, as $\alpha(t)=\alpha^{(2)}$ if $t \in\left[T_{1}, T_{1}+T_{2}[\right.$ and as $\alpha(t)=0$ if $t \in\left[T_{1}+T_{2}, T\right.$ [ and then extended by $T$-periodicity on the whole real line. Clearly, the same trick can be made on all the coefficients of (4.1), so that $(\mathbf{S})$ appears as an example of (4.1) with stepwise periodic coefficients. It might be interesting to investigate to what extent we can obtain a similar result for system (4.1) for more general $T$-periodic coefficients. From the proof of Theorem 4.1 we shall see that the result about chaotic dynamics is robust with respect to small perturbations of the Poincaré map. Technically, this claim follows by the same argument as in [34]. This fact can be then applied in order to extend the previous result to a broader class of coefficients in system (4.1) Namely, we have the following.

Theorem 4.2. Fix all the parameters in (S) satisfying the conditions of Theorem 4.1, i.e., assume (4.5), as well as $\gamma^{(2)}>\gamma_{2}^{*}$ and $T_{i}>T_{i}^{*}($ for $i=1,2,3)$. Then there exists $\epsilon>0$ such that if the distance in $L_{T}^{1}$ between the previous parameters in $(\mathbf{S})$ and the coefficients of (4.1) is smaller than $\epsilon$, the Poincaré map associated with (4.1) is chaotic.

Given two $T$-periodic integrable functions $f(t)$ and $g(t)$, their distance in $L_{T}^{1}$ is given by $\int_{0}^{T}|f(t)-g(t)| d t$. In our setting, the $\epsilon$-assumptions in Theorem 4.2 mean that

$$
\int_{0}^{T_{1}}\left|\alpha(t)-\alpha^{(1)}\right| d t+\int_{T_{1}}^{T_{1}+T_{2}}\left|\alpha(t)-\alpha^{(2)}\right| d t+\int_{T_{1}+T_{2}}^{T}|\alpha(t)| d t<\epsilon,
$$

and so on (for the other coefficients).

We observe that the functions satisfying conditions of form (4.6) are not necessary "piecewise constant" and they can be taken as smooth as we like.

We stress that, as a consequence of our definition of chaotic dynamics, under the assumptions of Theorem 4.2 we have infinitely many periodic solutions (subharmonics) for system (4.1). Indeed, the following result holds.

Corollary 4.1. Fix all the parameters in (S) satisfying the conditions of Theorem 4.1, i.e., assume (4.5), as well as $\gamma^{(2)}>\gamma_{2}^{*}$ and $T_{i}>T_{i}^{*}($ for $i=1,2,3)$. Then there exists $\epsilon>0$ such that if the distance in $L_{T}^{1}$ between the previous parameters in $(\mathbf{S})$ and the coefficients of (4.1) is smaller than $\epsilon$, system (4.1) has at least two T-periodic solutions as well as subharmonic solutions of any order with range in $\operatorname{int}\left(\mathbb{R}_{+}^{3}\right)$. 
To the best of our knowledge, it seems not an easy task to achieve multiplicity results for this kind of systems using functional analytic tools, due to the lack of a Hamiltonian structure for (4.1).

\section{Proofs}

We split the proof of Theorem 4.1 into four steps. In the first one we construct a topological parallelepiped (that is a set in int $\mathbb{R}_{+}^{3}$ homeomorphic to the unit cube) where the result of Sect. 3 is applied. In each of the three remaining steps we check the validity of the assumptions $\left(H_{f}\right),\left(H_{g}\right),\left(H_{h}\right)$ for the corresponding Poincaré maps.

Step 1: Definition of the parallelepiped.

First of all we consider the two-dimensional Lotka-Volterra system

$$
\left\{\begin{array}{l}
x_{1}^{\prime}=x_{1}\left(\alpha^{(1)}-a_{12}^{(1)} x_{2}\right) \\
x_{2}^{\prime}=x_{2}\left(-\beta^{(1)}+a_{21}^{(1)} x_{1}\right) .
\end{array}\right.
$$

As is well known, the equilibrium point $\left(\beta^{(1)} / a_{21}^{(1)}, \alpha^{(1)} / a_{12}^{(1)}\right)$ is a global center in the open first quadrant. Such a point is surrounded by a family of closed curves which are the level lines of the energy function

$$
\mathcal{E}\left(x_{1}, x_{2}\right):=a_{12}^{(1)} x_{1}-\alpha^{(1)} \log x_{1}+a_{21}^{(1)} x_{2}-\beta^{(1)} \log x_{2}
$$

satisfying

$$
\mathcal{E}\left(x_{1}, x_{2}\right)=k>\min \mathcal{E}=\mathcal{E}\left(\beta^{(1)} / a_{21}^{(1)}, \alpha^{(1)} / a_{12}^{(1)}\right) .
$$

An elementary argument, together with condition (4.5), enables us to deduce that there exists $\tilde{\ell}>0$ such that for all $0<\ell<\tilde{\ell}$ the following property holds.

The solution of (5.1) with initial point at $\left(\beta^{(1)} / a_{21}^{(1)}, \beta^{(2)} / 2\right)$ ) leaves the rectangle

$$
\left[\beta^{(1)} / a_{21}^{(1)}-\ell, \beta^{(1)} / a_{21}^{(1)}+\ell\right] \times\left[\beta^{(2)} / 4,3 \beta^{(2)} / 4\right]
$$

across the sides

$$
\left\{\beta^{(1)} / a_{21}^{(1)}-\ell\right\} \times\left[\beta^{(2)} / 4,3 \beta^{(2)} / 4\right] \text { and }\left\{\beta^{(1)} / a_{21}^{(1)}+\ell\right\} \times\left[\beta^{(2)} / 4,3 \beta^{(2)} / 4\right] .
$$

Analogously, the solution with initial point at $\left.\left(\beta^{(1)} / a_{21}^{(1)}, 3 \beta^{(2)} / 2\right)\right)$ leaves the rectangle

$$
\left[\beta^{(1)} / a_{21}^{(1)}-\ell, \beta^{(1)} / a_{21}^{(1)}+\ell\right] \times\left[5 \beta^{(2)} / 4,7 \beta^{(2)} / 4\right]
$$

across the sides

$\left\{\beta^{(1)} / a_{21}^{(1)}-\ell\right\} \times\left[5 \beta^{(2)} / 4,7 \beta^{(2)} / 4\right]$ and $\left\{\beta^{(1)} / a_{21}^{(1)}+\ell\right\} \times\left[5 \beta^{(2)} / 4,7 \beta^{(2)} / 4\right]$.

The standard proof is omitted. The interested reader can find more details in [33]. However, we observe that $\tilde{\ell}$ can be explicitly estimated. 


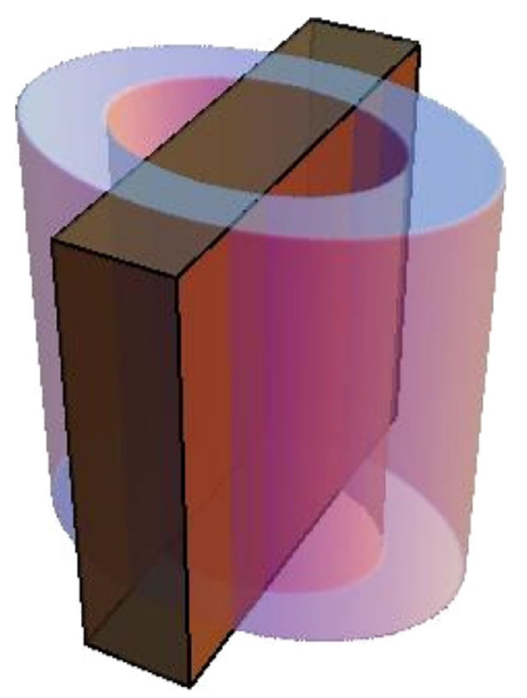

Figure 4. A pictorial description of $\mathcal{B}$

After this preliminary discussion, we can properly define the parallelepiped. To this aim, we introduce the cylindrical region $C y l \subset$ int $\mathbb{R}_{+}^{3}$ defined by

$\left\{\left(x_{1}, x_{2}\right): \mathcal{E}\left(x_{1}, x_{2}\right)=\mathcal{E}\left(\beta^{(1)} / a_{21}^{(1)}, x_{2}^{0}\right)\right.$ with $\left.x_{2}^{0} \in\left[\frac{\beta^{(2)}}{2}, \frac{3 \beta^{(2)}}{2}\right]\right\} \times\left[\frac{\beta^{(3)}}{2}, \frac{3 \beta^{(3)}}{2}\right]$.

Next, we fix a constant $\ell \in] 0, \tilde{\ell}[$ and define the set

$$
\mathcal{B}:=C y l \cap\left\{\left(x_{1}, x_{2}, x_{3}\right): x_{1} \in\left[\beta^{(1)} / a_{21}^{(1)}-\ell, \beta^{(1)} / a_{21}^{(1)}+\ell\right]\right\}
$$

(see Fig. 4 for a visualization of such a set).

By the choice of $\ell$ we know that $\mathcal{B}$ consists into exactly two connected components. Between these two components we select the one containing the point $\left(\beta^{(1)} / a_{21}^{(1)}, \beta^{(2)}, \beta^{(3)}\right)$. We denote by $\mathcal{P}$ such a component. By construction, we observe that the following inclusion holds

$$
\mathcal{M}:=\left[\frac{\beta^{(1)}}{a_{21}^{(1)}}-\ell, \frac{\beta^{(1)}}{a_{21}^{(1)}}+\ell\right] \times\left[\frac{3 \beta^{(2)}}{4}, \frac{5 \beta^{(2)}}{4}\right] \times\left[\frac{\beta^{(3)}}{2}, \frac{3 \beta^{(3)}}{2}\right] \subset \mathcal{P}
$$

(see also Fig. 5).

Our goal will be to prove that the set $\mathcal{P}$ contains a horseshoe for $\Phi$ under a suitable choice of times $T_{1}, T_{2}$, and $T_{3}$ using Theorem 3.1. In this proof the set $\mathcal{P}$ will play the role of the cube $R$, while the set corresponding to $R_{0}$ will be defined as a suitable subset $\mathcal{P}_{0}$ of the parallelepiped $\mathcal{M}$. The precise definition of $\mathcal{P}_{0}$ will be given in Step 3.

Step 2: Proof of the property $\left(H_{f}\right)$. 


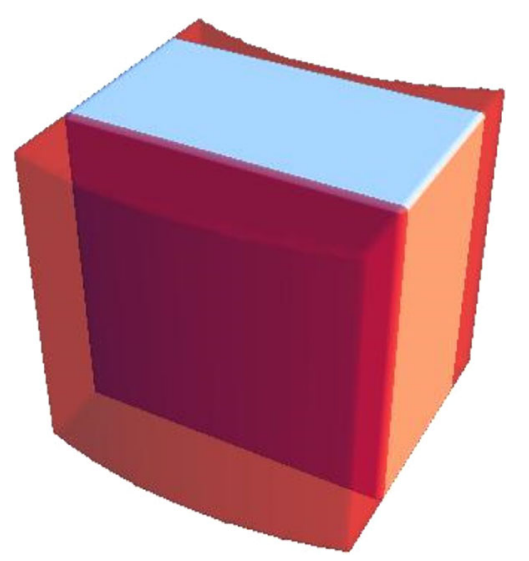

Figure 5. The set $\mathcal{P}$ with $\mathcal{M}$ inside

Consider the differential system

$$
\left\{\begin{array}{l}
x_{1}^{\prime}=x_{1}\left(\alpha^{(1)}-a_{12}^{(1)} x_{2}\right) \\
x_{2}^{\prime}=x_{2}\left(-\beta^{(1)}+a_{21}^{(1)} x_{1}\right) \\
x_{3}^{\prime}=0
\end{array}\right.
$$

The set of the equilibrium points in $\operatorname{int} \mathbb{R}_{+}^{3}$ for system (5.3) is given by the vertical line

$$
L:=\left\{\left(\frac{\beta^{(1)}}{a_{21}^{(1)}}, \frac{\alpha^{(1)}}{a_{12}^{(1)}}, x_{3}\right): x_{3}>0\right\} .
$$

Every horizontal plane $x_{3}=$ constant $>0$ is invariant for the solutions of system (5.3) and on this plane there is a global center at the point $\left(\frac{\beta^{(1)}}{a_{21}^{(1)}}, \frac{\alpha^{(1)}}{a_{12}^{(1)}}\right)$. Therefore, for any point $q_{0}:=\left(x_{1}^{0}, x_{2}^{0}, x_{3}^{0}\right) \notin L$ and for any $\tau>0$, we can define a rotation number in the following way:

$$
\operatorname{rot}\left(q_{0}, \tau\right):=\frac{1}{2 \pi} \int_{0}^{\tau} \frac{\left(x_{2}\left(t ; q_{0}\right)-\frac{\alpha^{(1)}}{a_{12}^{(1)}}\right) X_{1}(t)-\left(x_{1}\left(t ; q_{0}\right)-\frac{\beta^{(1)}}{a_{21}^{(1)}}\right) X_{2}(t)}{\left(x_{1}\left(t ; q_{0}\right)-\frac{\beta^{(1)}}{a_{21}^{(1)}}\right)^{2}+\left(x_{2}\left(t ; q_{0}\right)-\frac{\alpha^{(1)}}{a_{12}^{(1)}}\right)^{2}} d t
$$

where

$X_{1}(t):=x_{1}\left(t ; q_{0}\right)\left(\alpha^{(1)}-a_{12}^{(1)} x_{2}\left(t ; q_{0}\right)\right), \quad X_{2}(t):=x_{2}\left(t ; q_{0}\right)\left(-\beta^{(1)}+a_{21}^{(1)} x_{1}\left(t ; q_{0}\right)\right)$ and $\left(x_{1}\left(t ; q_{0}\right), x_{2}\left(t ; q_{0}\right), x_{3}^{0}\right)$ is the solution of system (5.3) departing at time $t=0$ from the initial point $q_{0}$.

The rotation number is an algebraic count of the number of turns around the equilibrium point in the plane $x_{3}=x_{3}^{0}$ during the time interval $[0, \tau]$. Since the trajectories of (5.3) wind counterclockwise in the $x_{1} x_{2}$-plane, the function $\tau \mapsto \operatorname{rot}\left(q_{0}, \tau\right)$ is strictly increasing for any $q_{0} \notin L$. 
To prove the stretching property $\left(H_{f}\right)$ we focus our attention on four "vertical" faces of $\mathcal{P}$. Namely, we define

$$
\begin{aligned}
\mathcal{P}^{\prime} & :=\left\{\left(x_{1}, x_{2}, x_{3}\right) \in \mathcal{P}: \mathcal{E}\left(x_{1}, x_{2}\right)=\mathcal{E}\left(\beta^{(1)} / a_{21}^{(1)}, \beta^{(2)} / 2\right)\right\}, \\
\mathcal{P}^{\prime \prime} & :=\left\{\left(x_{1}, x_{2}, x_{3}\right) \in \mathcal{P}: \mathcal{E}\left(x_{1}, x_{2}\right)=\mathcal{E}\left(\beta^{(1)} / a_{21}^{(1)}, 3 \beta^{(2)} / 2\right)\right\}
\end{aligned}
$$

and

$$
\begin{aligned}
& \mathcal{R}^{\prime}:=\left\{\left(x_{1}, x_{2}, x_{3}\right) \in \mathcal{P}: x_{1}=\beta^{(1)} / a_{21}^{(1)}-\ell\right\} \\
& \mathcal{R}^{\prime \prime}:=\left\{\left(x_{1}, x_{2}, x_{3}\right) \in \mathcal{P}: x_{1}=\beta^{(1)} / a_{21}^{(1)}+\ell\right\} .
\end{aligned}
$$

All the points of $\mathcal{P}^{\prime}$ (respectively, $\mathcal{P}^{\prime \prime}$ ) are periodic points of system (5.3) which share the same period. We denote by $T_{1}^{\prime}$ and by $T_{1}^{\prime \prime}$ the common periods for all the points of $\mathcal{P}^{\prime}$ and $\mathcal{P}^{\prime \prime}$, respectively. Since

$$
\mathcal{E}\left(\beta^{(1)} / a_{21}^{(1)}, \beta^{(2)} / 2\right)>\mathcal{E}\left(\beta^{(1)} / a_{21}^{(1)}, 3 \beta^{(2)} / 2\right),
$$

by the monotonicity of the period with respect to the energy level (see [40, Theorem 2]) we know that

$$
T_{1}^{\prime}>T_{1}^{\prime \prime}
$$

Now we fix

$$
T_{1}>T_{1}^{*}
$$

where

$$
T_{1}^{*}>\frac{5 T_{1}^{\prime} T_{1}^{\prime \prime}}{T_{1}^{\prime}-T_{1}^{\prime \prime}} .
$$

After that, we introduce the two integers $m^{\prime}$ and $m^{\prime \prime}$ which are, respectively, the largest integer smaller or equal than $T_{1} / T_{1}^{\prime}$ and the smallest integer greater or equal to $T_{1} / T_{1}^{\prime \prime}$. Accordingly, any point belonging to $\mathcal{P}^{\prime}$ makes at most $m^{\prime}$ turns and hence its associated rotation number is less than or equal to $\mathrm{m}^{\prime}$. Similarly, each point of the face $\mathcal{P}^{\prime \prime}$ performs at least $m^{\prime \prime}$ turns and has rotation number greater than or equal to $m^{\prime \prime}$. From (5.4) and the choice of $T_{1}$ it follows that the intervals $\left[m^{\prime}+1, m^{\prime}+2\right],\left[m^{\prime \prime}-2, m^{\prime \prime}-1\right]$, are disjoint.

Let us consider a system of cylindrical coordinates with reference to the vertical half-plane

$$
\sigma:=\left\{\left(x_{1}, x_{2}, x_{3}\right): x_{1}=\beta^{(1)} / a_{21}^{(1)}, x_{2}<\alpha^{(1)} / a_{12}^{(1)}\right\}
$$

and denote by $\vartheta\left(t, q_{0}\right)$ the angular coordinate of the solution $\left(x_{1}\left(t ; q_{0}\right), x_{2}\left(t ; q_{0}\right)\right.$, $x_{3}$ ) with initial point $q_{0} \in \mathcal{P}$. By the above properties of the rotation numbers,

$$
\vartheta\left(T_{1} ; q_{0}\right)<2 \pi m^{\prime}+\pi, \forall q_{0} \in \mathcal{P}^{\prime} \text { and } \vartheta\left(T_{1} ; q_{0}\right)>2 \pi m^{\prime}-\pi, \forall q_{0} \in \mathcal{P}^{\prime \prime} .
$$

Therefore the compact sets

$$
H_{0}:=\left\{q \in \mathcal{P}: \vartheta\left(T_{1} ; q\right) \in 2 \pi\left[m^{\prime}+1, m^{\prime}+2\right]\right\}
$$

and

$$
H_{1}:=\left\{q \in \mathcal{P}: \vartheta\left(T_{1} ; q\right) \in 2 \pi\left[m^{\prime \prime}-2, m^{\prime \prime}-1\right]\right\}
$$

are nonempty and disjoint. 
Our aim now is to prove the stretching property $\left(H_{f}\right)$, where we recall that $f$ is the Poincaré map associated with (5.3) for the interval $\left[0, T_{1}\right]$.

Let $\zeta=\left(\zeta_{1}, \zeta_{2}, \zeta_{3}\right):\left[t_{0}, t_{1}\right] \rightarrow \mathcal{P}$ be a path with $\zeta\left(t_{0}\right) \in \mathcal{P}^{\prime}$ and $\zeta\left(t_{1}\right) \in \mathcal{P}^{\prime \prime}$. Passing to the angular coordinates we consider the function

$$
\xi:\left[t_{0}, t_{1}\right] \ni s \mapsto \vartheta\left(T_{1} ; \zeta(s)\right) .
$$

By the above property of the angular function, we know that $\xi\left(t_{0}\right)<2 \pi m^{\prime}+\pi$ and $\xi\left(t_{1}\right)>2 \pi m^{\prime \prime}-\pi$. Using elementary continuity arguments we can define two subintervals $\left[s_{1}^{\prime}, s_{1}^{\prime \prime}\right],\left[s_{2}^{\prime}, s_{2}^{\prime \prime}\right]$ of $\left[t_{0}, t_{1}\right]$ such that $\zeta(s) \in H_{0}$ for every $s \in$ $\left[s_{1}^{\prime}, s_{1}^{\prime \prime}\right]$ and $\zeta(s) \in H_{1}$ for every $s \in\left[s_{2}^{\prime}, s_{2}^{\prime \prime}\right]$ and, moreover,

$$
f(\zeta(s))=\left(x_{1}\left(T_{1} ; \zeta(s)\right), x_{2}\left(T_{1} ; \zeta(s)\right), \zeta_{3}(s)\right) \in \mathcal{P}, \quad \forall s \in\left[s_{1}^{\prime}, s_{1}^{\prime \prime}\right] \cup\left[s_{2}^{\prime}, s_{2}^{\prime \prime}\right],
$$

with

$$
f\left(\zeta\left(s_{1}^{\prime}\right)\right), f\left(\zeta\left(s_{2}^{\prime}\right)\right) \in \mathcal{R}^{\prime} \quad \text { and } f\left(\zeta\left(s_{1}^{\prime \prime}\right)\right), f\left(\zeta\left(s_{2}^{\prime \prime}\right)\right) \in \mathcal{R}^{\prime \prime} .
$$

This concludes the proof of the second step.

Step 3: Proof of the property $\left(H_{g}\right)$.

First of all we define a set $\mathcal{P}_{0} \subset \mathcal{M}$ which will play the role of $R_{0}$ for Theorem 3.1. To this purpose, we consider the two-dimensional system in the $x_{1} x_{3}$-plane

$$
\left\{\begin{array}{l}
x_{1}^{\prime}=x_{1}\left(\alpha^{(2)}-a_{13}^{(2)} x_{3}\right) \\
x_{3}^{\prime}=x_{2}\left(-\gamma^{(2)}+a_{31}^{(2)} x_{3}\right) .
\end{array}\right.
$$

In such a system $\alpha^{(2)}, a_{13}^{(2)}$ and $a_{31}^{(2)}$ are fixed parameters. An elementary argument as in Step 1 says that there exists $\tilde{\gamma}_{2}$ such that for each $\gamma^{(2)}$ with

$$
\gamma^{(2)}>\gamma_{2}^{*}:=\max \left\{a_{31}^{(2)}\left(\beta^{(1)} / a_{21}^{(1)}+\ell\right), \tilde{\gamma}_{2}\right\}
$$

the following property holds: The solutions of (5.5) with initial point at $\left(\beta^{(1)} / a_{21}^{(1)}-\ell / 2, \beta^{(3)}\right)$ and at $\left(\beta^{(1)} / a_{21}^{(1)}-\ell / 2, \beta^{(3)}\right)$ leave the rectangle

$$
\left[\beta^{(1)} / a_{21}^{(1)}-\ell, \beta^{(1)} / a_{21}^{(1)}+\ell\right] \times\left[\beta^{(3)} / 2,3 \beta^{(2)} / 2\right]
$$

across the sides

$$
\begin{aligned}
& {\left[\beta^{(1)} / a_{21}^{(1)}-\ell, \beta^{(1)} / a_{21}^{(1)}+\ell\right] \times\left\{\beta^{(3)} / 2\right\} \text { and }} \\
& {\left[\beta^{(1)} / a_{21}^{(1)}-\ell, \beta^{(1)} / a_{21}^{(1)}+\ell\right] \times\left\{3 \beta^{(3)} / 2\right\} .}
\end{aligned}
$$

From now on, also assume that the parameter $\gamma^{(2)}$ is fixed, according to (5.6).

For the Hamiltonian system (5.5) we have the energy function

$$
\mathcal{F}\left(x_{1}, x_{3}\right):=a_{13}^{(2)} x_{1}-\alpha^{(2)} \log x_{1}+a_{31}^{(2)} x_{3}-\gamma^{(2)} \log x_{3}
$$

and, using $\mathcal{F}$, we can define the set $\mathcal{P}_{0}$ as the intersection of the parallelepiped $\mathcal{M}$ with the set

$$
\left\{\left(x_{1}, x_{2}, x_{3}\right): \mathcal{F}_{*} \leq \mathcal{F}\left(x_{1}, x_{3}\right) \leq \mathcal{F}^{*}\right\}
$$

where

$$
\mathcal{F}_{*}:=\mathcal{F}\left(\beta^{(1)} / a_{21}^{(1)}-\ell / 2, \beta^{(3)}\right), \quad \mathcal{F}^{*}:=\mathcal{F}\left(\beta^{(1)} / a_{21}^{(1)}+\ell / 2, \beta^{(3)}\right)
$$

(see also Fig. 6). 


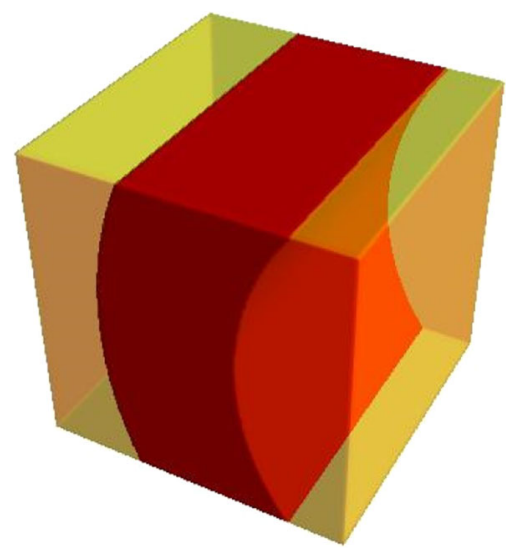

Figure 6. A pictorial description of the set $\mathcal{P}_{0}$

We also introduce the times $T_{2}^{\prime}$ and $T_{2}^{\prime \prime}$ which are the periods of the orbits passing through the points $\left(\beta^{(1)} / a_{21}^{(1)}-\ell / 2, \beta^{(3)}\right)$ and $\left(\beta^{(1)} / a_{21}^{(1)}+\ell / 2, \beta^{(3)}\right)$, respectively. Again by the monotonicity of the period with respect to the energy levels we know that

$$
T_{2}^{\prime}>T_{2}^{\prime \prime}
$$

We consider now the system

$$
\left\{\begin{array}{l}
x_{1}^{\prime}=x_{1}\left(\alpha^{(2)}-a_{13}^{(2)} x_{3}\right) \\
x_{2}^{\prime}=x_{2}\left(\beta^{(2)}-x_{2}\right) \\
x_{3}^{\prime}=x_{3}\left(-\gamma^{(2)}+a_{31}^{(2)} x_{1}\right)
\end{array}\right.
$$

for $t \in\left[0, T_{2}\right]$. With respect to the pair of variables $\left(x_{1}, x_{3}\right)$ we are in a situation analogous to system (5.1). Nevertheless, we have a compression effect toward the constant $\beta^{(2)}$ in the $x_{2}$-variable due to the logistic growth.

After these comments we properly concentrate on property $\left(H_{g}\right)$. Indeed, using the second equation in (5.8), we define a time $\tau_{2}>0$ such that $\mid x_{2}(t)-$ $\beta^{(2)} \mid \leq \beta^{(2)} / 4$ for all $t \geq \tau_{2}$ and any initial point in $\mathcal{P}$. Now we fix

$$
T_{2}>T_{2}^{*}
$$

where

$$
T_{2}^{*}>\max \left\{\frac{3 T_{2}^{\prime} T_{2}^{\prime \prime}}{T_{2}^{\prime}-T_{2}^{\prime \prime}}, \tau_{2}\right\} .
$$

Observe that $T_{2}^{*}=T_{2}^{*}\left(\gamma^{(2)}\right)$ because $T_{2}^{\prime}$ and $T_{2}^{\prime \prime}$ depend on $\gamma^{(2)}$.

Now we can check the stretching property $\left(H_{g}\right)$, where we recall that $g$ is the Poincaré map associated with (5.8) for the interval $\left[0, T_{2}\right]$, that is,

$$
g(q)=\left(y_{1}\left(T_{2} ; q\right), y_{2}\left(T_{2} ; q\right), y_{3}\left(T_{2} ; q\right)\right)
$$

where $\left(y_{1}(\cdot ; q), y_{2}(\cdot ; q), y_{3}(\cdot ; q)\right)$ denotes the solution of $(5.8)$ with initial value in $q$. 
Let $\zeta=\left(\zeta_{1}, \zeta_{2}, \zeta_{3}\right):\left[t_{0}, t_{1}\right] \rightarrow \mathcal{P}$ be a path with $\zeta\left(t_{0}\right) \in \mathcal{R}^{\prime}$ and $\zeta\left(t_{1}\right) \in$ $\mathcal{R}^{\prime \prime}$. As $T_{2}>\tau_{2}$ and $\zeta(s) \in \mathcal{P}$ for each $s \in\left[t_{0}, t_{1}\right]$, we know that $\mid y\left(T_{2} ; \zeta(s)\right)-$ $\beta^{(2)} \mid<\beta^{(2)} / 4$. On the other hand, there is a subinterval $\left[t_{0}^{\prime}, t_{1}^{\prime}\right] \subset\left[t_{0}, t_{1}\right]$ such that

$$
\mathcal{F}_{*} \leq \mathcal{F}\left(\zeta_{1}(s), \zeta_{2}(s)\right) \leq \mathcal{F}^{*}, \quad \forall s \in\left[t_{0}^{\prime}, t_{1}^{\prime}\right]
$$

with

$$
\mathcal{F}\left(\zeta_{1}\left(t_{0}^{\prime}\right), \zeta_{2}\left(t_{0}^{\prime}\right)\right)=\mathcal{F}_{*} \quad \text { and } \quad \mathcal{F}\left(\zeta_{1}\left(t_{1}^{\prime}\right), \zeta_{2}\left(t_{1}^{\prime}\right)\right)=\mathcal{F}^{*} .
$$

Moreover, by the definition of $\mathcal{P}$ we also deduce that

$$
\zeta_{3}(s) \in\left[\frac{\beta^{(3)}}{2}, \frac{3 \beta^{(3)}}{2}\right], \quad \forall s \in\left[t_{0}^{\prime}, t_{1}^{\prime}\right] .
$$

By introducing a system of polar coordinates in the $x_{1} x_{3}$-plane and arguing as in the previous step, we can find a subinterval $\left[s^{\prime}, s^{\prime \prime}\right] \subset\left[t_{0}^{\prime}, t_{1}^{\prime}\right]$ such that, for every $s \in\left[s^{\prime}, s^{\prime \prime}\right]$,

$\mathcal{F}_{*} \leq \mathcal{F}\left(y_{1}\left(T_{2} ; \gamma(s)\right), y_{3}\left(T_{2} ; \gamma(s)\right)\right) \leq \mathcal{F}^{*}, \quad$ and $\quad y_{3}\left(T_{2} ; \gamma(s)\right) \in\left[\frac{\beta^{(3)}}{2}, \frac{3 \beta^{(3)}}{2}\right]$

hold, with

$$
y_{3}\left(T_{2} ; \gamma\left(s^{\prime}\right)\right)=\frac{\beta^{(3)}}{2}, \quad y_{3}\left(T_{2} ; \gamma\left(s^{\prime \prime}\right)\right)=\frac{3 \beta^{(3)}}{2} .
$$

Collecting all the information about $\left(y_{1}\left(T_{2} ; q\right), y_{2}\left(T_{2} ; q\right), y_{3}\left(T_{2} ; q\right)\right)$ with $q$ in the sub-path of $\zeta$, we conclude that

$$
g(\zeta(s)) \in \mathcal{P}_{0}, \quad \forall s \in\left[s^{\prime}, s^{\prime \prime}\right]
$$

with $g\left(\zeta\left(s^{\prime}\right)\right)$ and $g\left(\zeta\left(s^{\prime \prime}\right)\right)$ belonging to the lower and the upper faces of $\mathcal{P}_{0}$, respectively. The proof of $\left(H_{g}\right)$ is thus completed.

Step 4: Proof of the property $\left(H_{h}\right)$.

As a last step we prove property $\left(H_{h}\right)$ with $\mathcal{P}$ and $\mathcal{P}_{0}$ playing the role of the sets $R$ and $R_{0}$ of the prototypical model and with $h$ the Poincaré map associated with system

$$
\left\{\begin{array}{l}
x_{1}^{\prime}=0 \\
x_{2}^{\prime}=x_{2}\left(\beta^{(3)}-x_{3}\right) \\
x_{3}^{\prime}=0
\end{array}\right.
$$

on the time interval $\left[0, T_{3}\right]$.

Let $\left(z_{1}(\cdot ; q), z_{2}(\cdot ; q), z_{3}(\cdot ; q)\right)$ denote the solution of $(5.10)$ with initial value in $q=\left(q_{1}, q_{2}, q_{3}\right)$. Since $z_{1}(t ; q)$ and $z_{3}(t ; q)$ are constant functions, the second equation in (5.10) reads as $x_{2}^{\prime}=x_{2}\left(\beta^{(3)}-q_{3}\right)$. Therefore, $x_{2}^{\prime}=-\frac{\beta^{(3)}}{2} x_{2}$ on the points of the upper face of $\mathcal{P}_{0}$ and $x_{2}^{\prime}=\frac{\beta^{(3)}}{2} x_{2}$ on the lower face. Now it is clear how to determine $T_{3}^{*}$ so that $\left(H_{h}\right)$ hods for every $T_{3}>T_{3}^{*}$.

This concludes the proof of Theorem 4.1.

To prove Theorem 4.2 it is sufficient to observe that all the results in the previous steps are stable with respect to small perturbations of the coefficients in the $L^{1}$-norm. Then our claim follows by repeating an argument already described in [34]. 
To be more specific, suppose that the parameters in $(\mathbf{S})$ as well as the constants $T_{1}>T_{1}^{*}, T_{2}>T_{2}^{*}$ and $T_{3}>T_{3}^{*}$ are fixed in order to have achieved the proof of Theorem 4.1 and consider now a system of the form (4.1) where the coefficients are so that in each interval $\left[0, T_{1}\right],\left[T_{1}, T_{1}+T_{2}\right]$ and $\left[T_{1}+T_{2}, T\right]$ they are $\epsilon$-close in the $L^{1}$-norm to those of the corresponding subsystems of (S). With this, we mean that (4.6) holds and hence $\int_{0}^{T_{1}}\left|\alpha(t)-\alpha^{(1)}\right| d t<\epsilon$, $\int_{T_{1}}^{T_{1}+T_{2}}\left|\alpha(t)-\alpha^{(2)}\right| d t<\epsilon, \int_{T_{1}+T_{2}}^{T}|\alpha(t)| d t<\epsilon$ (and so on the for the other coefficients). Now, if we check the argument in the proof for (S) of Theorem 4.1 it is easy to recognize that the verification of the three steps $\left(H_{f}\right),\left(H_{g}\right)$ and $\left(H_{h}\right)$ are based on obtaining strict inequalities (either on the rotation numbers or on some components of the partial Poincaré maps) which are still true if we consider a sufficiently small perturbation in the coefficients (by the theorem of continuous dependence of the solutions for Carathéodory systems). In this manner, we can guarantee that the conclusion of Theorem 4.1 extends to non-autonomous systems which are sufficiently small perturbations of the stepwise autonomous system (S).

\section{Acknowledgments}

The first author acknowledges the kind hospitality of the Department of Mathematics and Computer Science of the University of Udine.

\section{References}

[1] Ahmad, S., Lazer, A.C.: Average growth and extinction in a competitive LotkaVolterra system. Nonlinear Anal. 62, 545-557 (2005)

[2] Arioli, G., Zgliczyński, P.: Symbolic dynamics for the Hénon-Heiles Hamiltonian on the critical level. J. Differ. Equ. 171, 173-202 (2001)

[3] Block, L.S., Coppel, W.A.: Dynamics in One Dimension, Lecture Notes in Mathematics, vol. 1513. Springer, Berlin, New York (1992)

[4] Burns, K., Weiss, H.: A geometric criterion for positive topological entropy. Commun. Math. Phys. 172, 95-118 (1995)

[5] Capietto, A., Dambrosio, W., Papini, D.: Superlinear indefinite equations on the real line and chaotic dynamics. J. Differ. Equ. 181, 419-438 (2002)

[6] Carbinatto, M., Kwapisz, J., Mischaikow, K.: Horseshoes and the Conley index spectrum. Ergod. Theory Dyn. Syst. 20, 365-377 (2000)

[7] Cushing, J.M.: Periodic time-dependent predator-prey systems. SIAM J. Appl. Math. 32, 82-95 (1977)

[8] Cushing, J.M.: Periodic two-predator, one-prey interactions and the time sharing of a resource niche. SIAM J. Appl. Math. 44, 392-410 (1984) 
[9] DuBowy, P.J.: Waterfowl communities and seasonal environments: temporal variability in interspecific competition. Ecology 69, 1439-1453 (1988)

[10] Gaines, R.E., Mawhin, J.L.: Coincidence Degree, and Nonlinear Differential Equations, Lecture Notes in Mathematics, vol. 568. Springer, Berlin, New York (1977)

[11] Guckenheimer, J., Holmes, P.: Nonlinear Oscillations, Dynamical Systems, and Bifurcations of Vector Fields, Revised and Corrected Reprint of the 1983 Original. Applied Mathematical Sciences, 42. Springer, New York (1990)

[12] Hsu, S.-B., Zhao, X.-Q.: A Lotka-Volterra competition model with seasonal succession. J. Math. Biol. 64, 109-130 (2012)

[13] Hu, S.S., Tessier, A.J.: Seasonal succession and the strength of intra- and interspecific competition in a Daphnia assemblage. Ecology 76, 2278-2294 (1995)

[14] Huppert, A., Blasius, B., Olinky, R., Stone, L.: A model for seasonal phytoplankton blooms. J. Theor. Biol. 236, 276-290 (2005)

[15] Keeling, M., Rohani, P., Grenfell, P., B.T.: Seasonally forced disease dynamics explored as switching between attractors. Physica D 148, 317-335 (2001)

[16] Kennedy, J., Koçak, S., Yorke, J.A.: A chaos lemma. Am. Math. Mon. 108, 411$423(2001)$

[17] Kennedy, J., Yorke, J.A.: Topological horseshoes. Trans. Am. Math. Soc. 353, 2513-2530 (2001)

[18] Kennedy, J., Yorke, J.A.: Generalized Hénon difference equations with delay. Univ. Iagel. Acta Math. 41, 9-28 (2003)

[19] Kirchgraber, U., Stoffer, D.: On the definition of chaos. Z. Angew. Math. Mech. 69, 175-185 (1989)

[20] Krasnosel'skiǔ, M.A.: The Operator of Translation Along the Trajectories of Differential Equations, Translations of Mathematical Monographs, vol. 19. American Mathematical Society, Providence (1968)

[21] Krasnosel'skiı̌ M.A., Zabreı̌ko, P.P.: Geometrical methods of nonlinear analysis, Grundlehren der Mathematischen Wissenschaften 263, Springer, Berlin (1984)

[22] Krikorian, N.: The Volterra model for three species predator-prey systems: boundedness and stability. J. Math. Biol. 7, 117-132 (1979)

[23] Lisena, B.: Asymptotic behaviour in periodic three species predator-prey systems. Ann. Mat. Pura Appl. 186, 85-98 (2007)

[24] Malik, T., Smith, H.L.: Does dormancy increase fitness of bacterial populations in time-varying environments?. Bull. Math. Biol. 70, 1140-1162 (2008)

[25] Mawhin, J.: Topological Degree Methods in Nonlinear Boundary Value Problems, CBMS Regional Conference Series in Mathematics, 40. American Mathematical Society, Providence (1979) 
[26] Mawhin, J.: The legacy of Pierre-François Verhulst and Vito Volterra in population dynamics. In: The first 60 years of nonlinear analysis of Jean Mawhin, pp. 147-160. World Science Publications, River Edge (2004)

[27] Medio, A., Pireddu, M., Zanolin, F.: Chaotic dynamics for maps in one and two dimensions: a geometrical method and applications to economics. Int. J. Bifur. Chaos Appl. Sci. Eng. 19, 3283-3309 (2009)

[28] Moser, J.: Stable and random motions in dynamical systems. With Special Emphasis on Celestial Mechanics, Hermann Weyl Lectures, the Institute for Advanced Study, Princeton, N. J. Annals of Mathematics Studies, No. 77. Princeton University Press, Princeton (1973)

[29] Papini, D., Zanolin, F.: On the periodic boundary value problem and chaotic-like dynamics for nonlinear Hill's equations. Adv. Nonlinear Stud. 4, 71-91 (2004)

[30] Papini, D., Zanolin, F.: Some results on the periodic points and chaotic dynamics arising from the study of the nonlinear Hill equation. Rend. Sem. Mat. Univ. Pol. Torino (Subalpine Rhapsody in Dynamics) 65, 115-157 (2007)

[31] Pireddu, M., Zanolin, F.: Cutting surfaces and applications to periodic points and chaotic-like dynamics. Topol. Methods Nonlinear Anal. 30, 279-319 (2007); Correction in Topol. Methods Nonlinear Anal. 33, 395 (2009)

[32] Rouche, N., Mawhin, J.: Équations différentielles ordinaires. Tome II: Stabilité et solutions périodiques. Masson et Cie, Paris (1973)

[33] Ruiz-Herrera, A.: Chaos in predatorprey systems with/without impulsive effect. Nonlinear Anal. Real World Appl. 13, 977-986 (2012)

[34] Ruiz-Herrera, A., Zanolin, F.: An example of chaotic dynamics in 3D systems via stretching along paths. Ann. Mat. Pura Appl. 193, 163-185 (2014)

[35] Smale, S. Diffeomorphisms with many periodic points. In: Differential and Combinatorial Topology (A Symposium in Honor of Marston Morse) pp. 63-80. Princeton University Press, Princeton (1965)

[36] Smale, S.: Differentiable dynamical systems. Bull. Am. Math. Soc. 73, 747$817(1967)$

[37] Smale, S.: Finding a horseshoe on the beaches of Rio. Math. Intell. 20, 39$44(1998)$

[38] Srzednicki, R., Wójcik, K., Zgliczyński, P.: Fixed point results based on the Ważewski method, In: Brown, R.F., Furi, M., Górniewicz, L., Jiang, B. (eds.) Handbook of Topological Fixed Point Theory, pp. 905-943. Springer, Dordrecht (2005)

[39] Volterra, V.: Variazioni e fluttuazioni del numero d'individui in specie animali conviventi. Memorie R. Accad. Lincei 6(2), 31-113 (1926)

[40] Waldvogel, J.: The period in the Lotka-Volterra system is monotonic. J. Math. Anal. Appl. 114, 178-184 (1986) 
[41] Wójcik, K.: Remark on complicated dynamics of some planar system. J. Math. Anal. Appl. 271, 257-266 (2002)

[42] Zgliczyński P.: Fixed point index for iterations of maps. topological horseshoe and chaos. Topol. Methods Nonlinear Anal. 8, 169-177 (1986)

[43] Zgliczyński, P., Gidea, M.: Covering relations for multidimensional dynamical systems. J. Differ. Equ. 202, 32-58 (2004)

Alfonso Ruiz-Herrera

Departamento de Matemática Aplicada II

Universidade de Vigo

36310 Vigo

Spain

e-mail: alfonsoruiz@dma.uvigo.es

Fabio Zanolin

Department of Mathematics and Computer Science

University of Udine

via delle Scienze 206

33100 Udine

Italy

e-mail: fabio.zanolin@uniud.it

Received: 15 April 2014.

Revised: 9 December 2014.

Accepted: 11 December 2014. 